\begin{tabular}{|c|c|}
\hline \multicolumn{2}{|c|}{$\begin{array}{c}\text { Advertisers in This Issue } \\
\text { Page No. }\end{array}$} \\
\hline${ }^{*}$ American Institute of Physics & 94 \\
\hline Annual Reviews & 41 \\
\hline ASM International & 11 \\
\hline Australian Scientific Instruments & 6 \\
\hline CDS Analytical & 8 \\
\hline Cerac Inc. & 72 \\
\hline${ }^{\star}$ Digital Instruments & 7 \\
\hline${ }^{\star}$ EDAX International Inc. & 24 \\
\hline General Microdevices, Inc. & 8 \\
\hline Gordon Research Conference & 81 \\
\hline *Heraeus Amersil, Inc. & 23 \\
\hline${ }^{\star}$ High Voltage Engineering & ront cover \\
\hline * Huntington Laboratories & ack cover \\
\hline * JEOL USA, Inc. & 15 \\
\hline *Lambda Technologies & 23 \\
\hline * MDC Vacuum Products Corp. & 10 \\
\hline The Mellen Company, Inc. & 22 \\
\hline Microscopy Courses & 23 \\
\hline NanoPowder Enterprises Inc. & 29 \\
\hline *National Electrostatics Corp. & 24 \\
\hline${ }^{\star}$ New Focus, Inc. & ack cover \\
\hline Oxford Cryosystems & 18 \\
\hline *Park Scientific Instruments & 2 \\
\hline *Parke Mathematical Laboratories & 13 \\
\hline *Philips Analytical X-Ray & 1 \\
\hline Princeton Gamma-Tech, Inc. & 75 \\
\hline Quantum Design & 17 \\
\hline Shimadzu Corp. & 16 \\
\hline Structured Materials Industries, Inc. & 81 \\
\hline${ }^{\star}$ Tencor Instruments & 35 \\
\hline University of California, Berkeley & 99 \\
\hline ”Virginia Semiconductor, Inc. & 9,78 \\
\hline VLSI Standards & 74 \\
\hline *Voltaix, Inc. & 75 \\
\hline John Wiley \& Sons, Inc. & 48 \\
\hline $\begin{array}{l}\text { For free information about the products } \\
\text { vices offered in this issue, fill out and ma } \\
\text { Reader Service Card, or FAX it to (312) }\end{array}$ & $\begin{array}{l}\text { Ind ser- } \\
\text { il the } \\
22-3165 \text {. }\end{array}$ \\
\hline *Exhibiting at the 1997 MRS Spring M & \\
\hline
\end{tabular}

\title{
MRS Invites Nominations for the Von Hippel Award, MRS Medal, and Turnbull Lectureship
}

The Materials Research Society is seeking nominations for the Von Hippel Award, the MRS Medal, and the Turnbull Lectureship. The deadline for nominations is June 1, 1997. These awards will be presented at the 1997 MRS Fall Meeting, December 1-5, in Boston.

The MRS Awards Program recognizes outstanding contributors to the progress of materials research. Nomination forms and details about eligibility and nomination criteria are available from Anne Wagner, Materials Research Society, 9800 McKnight Road, Pittsburgh, PA 152376006; phone 412-367-3004 ext. 102; fax 412-367-4373. Further information about each award can also be obtained from MRS homepage on the World Wide Web: http://www.mrs.org.

\section{Von Hippel Award Acknowledges Outstanding Interdisciplinary Work in Materials Research}

The Von Hippel Award, first presented to Arthur R. von Hippel whose interdisciplinary and pioneering research typified the spirit of the award, is the Materials Research Society's highest honor. The recipient is recognized for brilliance and originality combined with vision that transcends the boundaries of conventional scientific disciplines. The Award includes a $\$ 10,000$ cash prize, honorary membership in MRS, and a unique trophy - a mounted ruby laser crystal symbolizing the many-faceted nature of materials research.

Selection of the recipient is determined by a vote of the MRS Council. The recipi- ent will be invited to speak at the Awards Ceremony.

\section{Turnbull Lectureship Honors Careèr of an Outstanding} Researcher and Communicator

The David Turnbull Lectureship recognizes the career of a scientist who has made outstanding contributions to understanding materials phenomena and properties through research, writing, and lecturing, as exemplified by the life work of David Turnbull. While honoring the accomplishments of the recipient, the Turnbull Lectureship is intended to support and enrich the materials research community.

The recipient will give a technical lecture of broad appeal at a designated session of the 1997 MRS Fall Meeting. The Turnbull Lecturer will receive a $\$ 5,000$ honorarium and a citation plaque, along with travel expenses paid to enable the recipient to address MRS Sections and University Chapters, and/or participate in the production of a video version of the lecture.

\section{MRS Medal Recognizes Recent Discovery or Advancement in Materials Science}

The MRS Medal offers public and professional recognition of an exceptional recent achievement in materials research. A medal will be awarded for a specific outstanding recent discovery or advancement which is expected to have a major impact on the progress of any materials-related field.

The award consists of a $\$ 3,000$ cash prize, an engraved and mounted medal, and a citation certificate.

\section{MRS Spring Meeting} Outstanding Young Investigator Iward:
Ohristopher I. Bowmintill, Lniversity of Colorado

"Polymmerization and Properties of Polymer Stabilized Ferroelectric Liquid l'rystals" Wednesday, Lpril 2, 5:00 p.II.

Golden Gate Al, San Prancisco Marrioull 


\section{Materials Research Society would like to thank the following for their financial support:}

ABB Extrel

Advanced Control Systems Corporation

Advanced Energy Industries, Inc.

Advanced Micro Devices, Inc.

Aerospace Corporation

Aetrium, Inc.

AG Associates

Air Products and Chemicals, Inc.

AIXTRON, Inc.

AKZO Nobel Chemicals, Inc.

Aldrich Chemical Company, Inc.

Aluminum Company of America

Ames Laboratory

AMRAY, Inc.

APD Cryogenics, Inc.

Applied Materials/Implant Division

ARCO Chemical Company

Argonne National Laboratory

Asahi Glass Co., Ltd.

Ashland Inc.

ASM Epitaxy

AST elektronik GmbH

ASTeX/Applied Science and

Technology, Inc.

Battelle Pacific Northwest Labs

Bayer Corporation/Bayer AG

Bede Scientific Incorporated

Bell Laboratories, Lucent Technologies

Blake Industries, Inc.

BP International Limited

British Nuclear Fuels

Brookhaven Instruments Corp.

Bruker Instruments, Inc.

Cameca Instruments, Inc.

Canon, Inc.

CGS Thermodynamics

Chemat Technology, Inc.

Chichibu Onoda Cement Co., Ltd.

Chisso Corp.

CNR-IMETEM

CNRS

Coherent, Inc./Laser Group

Commonwealth Scientific Corporation

Conductus, Inc.

Consortium für Elektrochemische

Industrie $\mathrm{GmbH}$

Continental Electronics Corporation

Co.Ri.M.Me

Cree Research, Inc.

Criterion Catalyst Company L.P.

Current Sciences Group

CVC Products, Inc.

Cymer Laser Technologies

Dainippon Screen Mfg. Co., Ltd.

DCA Instruments, Inc.

Denton Vacuum, Inc.

Diamond Semiconductor Group, Inc.

Diatome U.S.

Digital Equipment Corporation

Digital Instruments, Inc.

Dow Corning Corporation

dpiX, a Xerox Company

DSM Research

Dubbeldee Harris Diamond Corp.

Dupont Company, Central Research and Development

Dytech International

Eastman Kodak Company

Eaton Corporation

EDAX International

EG\&G Instruments, Inc

EG\&G Ortec

Electric Power Research Institute (EPRI)

Elsevier Science

EMCORE Corporation

Energy Conversion Devices

ESM Software
Charles Evans \& Associates

Evans East

Exxon Production Research Company

Exxon Research and Engineering

Company

FEl Company

E.A. Fischione Instruments, Inc.

Ford Motor Company

Forschungszentrum Rossendorf e.V.

FSI International

Fuji Electric Co., Ltd.

Fujjikin of America, Inc.

Furukawa Electric Co., Ltd

Gatan, Inc.

Gelest, Inc.

Genentech, Inc.

General Motors R\&D

GMW Associates

Goodfellow Corporation

Gordon and Breach

Gould Electronics Inc.

POWERDEX Division

Granville-Phillips Company

Hewlett Packard Company

Optoelectronics Division

Hewlett Packard, NMD

High Voltage Engineering

Hitachi Scientific Instruments

Hoechst Celanese Advanced

Technology Group

Hughes Research Laboratories

Huntington Mechanical Laboratories, Inc.

Hysitron, Inc.

IBM Analytical and Test Services

IBM, Thomas J. Watson

Research Center

Inorgtech, Ltd.

Institut für Schicht und lonentechnik (ISI)

Institute for Scientific Information (ISI)

Instron Corporation

Intel Corporation

Ion Tech, Inc.

ITAC, Ltd.

JCPDS-International Centre for

Diffraction Data

JEOL USA, Inc.

Johnsen Ultravac

Johnson Controls, Inc.

Johnson \& Johnson

Johnson \& Johnson Professional, Inc.

Johnson Matthey Electronics, Inc.

k-Space Associates, Inc.

Keithley Instruments, Inc.

Kimball Physics, Inc.

Kobe Steel USA, Inc.

Electronic Materials Center

Komag, Inc.

Kratos Analytical, Inc.

Lake Shore Cryotronics, Inc

Lambda Physik, Inc.

Lambda Technologies

Lawrence Berkeley National Laboratory

Lawrence Livermore National Laboratory

LEO Electron Microscopy Inc.

Kurt J. Lesker Company

Leybold Vacuum Products, Inc.

Los Alamos National Laboratory

Materials Research Corporation

Materials Research Group, Inc.

Mattson Technology, Inc.

MDC Vacuum Products Corporation

MEL Chemicals

Micro Photonics, Inc.

Microwave Research Center

Millipore Corporation

Mitsubishi Materials Corporation
Mitsui-Toatsu Chemicals, Inc.

MKS Instruments, Inc.

MMR Technologies, Inc

Mobil Technology Co.

Molecular Simulations, inc.

Morton International, Metalorganics

Motorola APRDL

MR Semicon, Inc.

MVSystems, Inc.

n\&k Technology, Inc.

Nanophase Technologies Corporation

National Electrostatics Corporation

National Renewable Energy Laboratory (NREL)

National Semiconductor Corporation

National Technology Transfer Center

NEC Corporation

NEC Research Institute, Inc.

Neocera, Inc.

New Focus, Inc.

Niki Glass Company, Inc.

Nikko Hitech International, Inc.

Nippon Steel Corporation

Nor-Cal Products, Inc.

Northrop Grumman

Science \& Technology Center

Oak Ridge National Laboratory

Omicron Associates

OnTrak Systems, Inc.

Osmic, Inc.

Oxford Cryosystems

Oxford Instruments, Inc.

Park Scientific Instruments

Parke Mathematical Laboratories

Pfeiffer Vacuum Technology, Inc.

Philips Electron Optics

Philips Electronic Instruments Co. (PEI)

Philips Semiconductors

Physical Electronics, Inc. (PHI)

PIOLAX Incorporated

Plasma Sciences, Inc.

Plasma-Therm, Inc.

Plenum Publishing $\mathrm{Co}$

Portland Cement Association

President Enterprises Co.

Princeton Gamma-Tech, Inc.

Princeton Measurements Corporation

Pure Tech, Inc.

Quantum Design, Inc.

Research and PVD Materials Corporation

RHK Technology, Inc.

Riber Division/ISA Inc.

Rigaku/USA, Inc.

RIMCOF (R\&D Institute of Metals \&

Composites for Future Industries)

RJ Lee Instruments, Ltd.

Rockwell International Science Center

Rohm and Haas Company

Samsung Advanced Institute of

Technology

Sandia National Laboratories

Santa Clara Plastics

Sanyo Electric Co., Ltd.

Schering Plough Research Institute

Schlumberger Cambridge Research

Schumacher, Unit of Air Products \&

Chemicals

Screen DNS Electronics

SEH America, Inc.

Sematech, Inc.

Semiconductor Processing $\mathrm{Co}_{0}$

Semitool, Inc.

SensArray Corporation

Sharp Corporation

Sharp Microelectronics Technology, Inc.

Shell Chemical Company

Shin-Etsu Handotai Co., Ltd.

Siemens AG

Siemens Energy \& Automation, Inc.

Analytical Instrumentation

Siltec Silicon

Smith \& Nephew Research, Ltd.

Soitec USA, Inc.

Solarex Corporation

Solartron Instruments

SONY Corporation

South Bay Technology, Inc

Springer-Verlag New York, Inc.

STAIB Instruments, Inc.

Strem Chemicals, Inc.

SubMicron Systems, Inc.

SULA Technologies

Sumitomo Electric USA, Inc.

Sumitomo Sitix Corporation

Supelec (a.k.a. Electricite de France)

SURFACE

Surface/Interface, Inc

SVT Associates

Taisil Electronic Materials Corp

Taiwan Four Pillars Enterprise Co., Ltd

Taiwan TASCO Chemical Corporation

Technology Modeling Associates, Inc.

Telefunken Microelectronic

Telemark

Tencor Instruments

Texas Instruments, Inc.

Thermionics Laboratory, Inc.

$3 \mathrm{M}$ Industrial and Consumer Sector

Research Laboratory

TOPCON Technologies, Inc.

TopoMetrix Corp.

Tosoh SMD, Inc.

Union Carbide Corporation

United Microelectronics Corporation

United Solar Systems Corporation

Universal Display Corporation

UOP Research Center

US Inc.

Vacuum Science Instruments, Inc. (VSI)

Vanguard international

Semiconductor Corp.

Varian Associates, Inc.

VCR Group, Inc.

VG Scientific

Virginia Semiconductor, Inc.

Virtual Laboratories

Voltaix, Inc.

Vortek Industries, Ltd.

VSP_International Science Publishers

UNIAX

Wacker Siltronic AG

John Wiley \& Sons, Inc.

W.R. Grace \& Company

Xerox Corporation 